\title{
ARTICLE
}

Received 15 May 2015 | Accepted 29 Jul 2015 | Published 14 Sep 2015

DOI: $10.1038 /$ ncomms 9206

\section{Nanophotonic coherent light-matter interfaces based on rare-earth-doped crystals}

Tian Zhong ${ }^{1}$, Jonathan M. Kindem ${ }^{1}$, Evan Miyazono ${ }^{1} \&$ Andrei Faraon $^{1}$

Quantum light-matter interfaces connecting stationary qubits to photons will enable optical networks for quantum communications, precise global time keeping, photon switching and studies of fundamental physics. Rare-earth-ion-doped crystals are state-of-the-art materials for optical quantum memories and quantum transducers between optical photons, microwave photons and spin waves. Here we demonstrate coupling of an ensemble of neodymium rare-earth-ions to photonic nanocavities fabricated in the yttrium orthosilicate host crystal. Cavity quantum electrodynamics effects including Purcell enhancement $(F=42)$ and dipole-induced transparency are observed on the highly coherent ${ }^{4} I_{9 / 2}{ }^{4} F_{3 / 2}$ optical transition. Fluctuations in the cavity transmission due to statistical fine structure of the atomic density are measured, indicating operation at the quantum level. Coherent optical control of cavity-coupled rare-earth ions is performed via photon echoes. Long optical coherence times $\left(T_{2} \sim 100 \mu \mathrm{s}\right)$ and small inhomogeneous broadening are measured for the cavity-coupled rare-earth ions, thus demonstrating their potential for on-chip scalable quantum light-matter interfaces.

\footnotetext{
${ }^{1}$ T. J. Watson Laboratory of Applied Physics, California Institute of Technology, 1200 East California Boulevard, MC 107-81, Pasadena, California 91125, USA
} Correspondence and requests for materials should be addressed to A.F. (email: faraon@caltech.edu). 
O uantum light-matter interfaces (QLMIs) are quantum devices composed of light emitters with quantum states that can be controlled via optical fields and entangled to photons $^{1,2}$. They enable distribution of quantum entanglement over long distances in optical quantum networks for quantum communications ${ }^{1}$. Quantum networks of atomic clocks have also been proposed for precise global time keeping and studies of fundamental physics ${ }^{2}$. Realizing QLMIs requires control of light and matter at the single atom and single photon level, which enables optoelectronic devices such as optical modulators and nonlinear optical devices operating at the most fundamental $\operatorname{level}^{3}$. QLMIs are also expected to play a leading role in realizing optical to microwave quantum transducers for interconnecting future superconducting quantum machines via optical fibres ${ }^{4,5}$.

Scalable and robust QLMIs require emitters to have long spin coherence times and coherent optical transitions. For integrated optical quantum networks, these emitters need to be coupled to on-chip optical resonators that capture the photons in a single mode and further couple them into optical fibres or waveguides. The solid-state emitters most investigated so far for on-chip QLMIs are semiconductor quantum dots (QDs) ${ }^{6}$ and nitrogen vacancy $(\mathrm{NV})$ centres in diamond ${ }^{7}$. To date, complete quantum control of single QD and NV spins, spin-photon entanglement and entanglement of remote NVs via photons have been realized $^{8-10}$. Both QDs ${ }^{11}$ and $\mathrm{NVs}^{12}$ have been coupled to optical nanocavities. However, the challenge in growing optically identical QDs limits their prospects for a scalable architecture ${ }^{6}$. NVs embedded in nanostructures have long electronic spin coherence times ${ }^{13}$, but suffer from optical spectral instabilities such as blinking and spectral diffusion ${ }^{14}$. These spectral instabilities have so far impeded the coherent coupling between optical fields and NV centres in nanoresonators that are essential for further developments of QLMIs.

Rare-earth ions (REIs) embedded in host crystals at cryogenic temperatures exhibit highly coherent quantum states in the $4 f$ orbital $^{15}$. The Zeeman or hyperfine states of REIs can have coherence times as long as 6 hours $^{16}$, the longest ever demonstrated in a solid. These states are connected via optical transitions with the narrowest linewidth in the solid state (sub$\mathrm{kHz}$ ) and small inhomogeneous broadening ( $\mathrm{MHz}$ to $\mathrm{GHz})^{17}$. This outstanding optical and spin coherence makes REI-doped crystals the state-of-the-art material for macroscopic solid-state optical quantum memories ${ }^{18,19}$. Integrated REI-doped waveguide quantum memories have also been developed ${ }^{20,21}$. Detection and control of single REI spins has been recently demonstrated in bulk material, but not using the transitions employed in optical quantum memories at cryogenic temperatures ${ }^{22,23}$. Coupling the highly coherent optical transitions of REIs to nanocavities will enable on-chip QLMIs, where REI ensembles act as quantum memories and single REIs act as qubits ${ }^{24}$.

Here we demonstrate high-cooperativity coupling of a neodymium $\left(\mathrm{Nd}^{3+}\right)$ ensemble to photonic nanocavities fabricated directly in the yttrium orthosilicate (YSO) host crystal and show coherent optical control of REIs coupled to nanophotonic cavities. These results are enabled by the long coherence time and small inhomogeneous broadening of cavitycoupled REIs, which are essential properties that may lead to nanophotonic QLMIs with better prospects for scalability than those based on NVs and QDs.

\section{Results}

Photonic nanocavities in YSO. The nanocavities, one of which is shown in Fig. 1a, were fabricated in neodymium-doped YSO $\left(\mathrm{Nd}^{3+}\right.$ :YSO) using focused ion beam milling. For this study, we used devices fabricated in two types of samples with $\mathrm{Nd}$ doping of
$0.2 \%$ and $0.003 \%$ (Scientific Materials Inc.). The photonic crystal cavity is made of grooves milled in a triangular nanobeam ${ }^{25}$ (Fig. 1b) (see Methods). Finite-difference time-domain simulations ${ }^{26}$ indicate a transverse electric (TE) mode with quality factor exceeding $1 \times 10^{5}$, mode volume $V_{\text {mode }}=1.65(\lambda)$ $\left.n_{\mathrm{YSO}}\right)^{3}=0.2 \mu \mathrm{m}^{3}$ and mode profile shown in Fig. $1 \mathrm{~b}$. Here $V$ is defined as $V_{\text {mode }}=\int_{V} \epsilon(\mathbf{r})|\mathrm{E}(\mathbf{r})|^{2} \mathrm{~d}^{3} \mathbf{r} / \max \left(\epsilon(\mathbf{r})|\mathrm{E}(\mathbf{r})|^{2}\right)$, where $\mathrm{E}(\mathbf{r})$ is the electric field and $\epsilon(\mathbf{r})$ is the electric permittivity at position r. Two $45^{\circ}$ angled cuts at both ends of the nanobeam (that is, couplers) allow for coupling light from a direction normal to the chip (that is, b in Fig. 1b) using a confocal microscope setup (see Methods). A broadband light source was coupled into the resonator from one end and the transmitted light was collected from the other coupler with typical efficiencies ranging from $20 \%$ to $50 \%$. The transmitted spectrum shows a resonance with quality factor $Q=4,400$ (Fig. 1c) in the device used for the following measurements. Arrays of devices were reproducibly fabricated with similar performance (Supplementary Fig. 1 and Supplementary Note 1).

Coupling rate between REIs and the nanocavity. The coupling of $\mathrm{Nd}^{3+}$ ions to the nanocavity was observed through enhancement in photoluminescence (PL) and emission rates. With the $0.2 \%$ device cooled at $3.5 \mathrm{~K}$ (Montana Instruments cryostation), an $810 \mathrm{~nm}$ laser coupled into the cavity excited PL in the ${ }^{4} \mathrm{I}_{9 / 2}-{ }^{4} \mathrm{~F}_{3 / 2}$ transition that was then collected from the output coupler (Fig. 2a). The PL spectrum shows two lines at 883.05 and $884.06 \mathrm{~nm}$, corresponding to two inequivalent sites $\left(Y_{1}\right.$ and $\left.Y_{2}\right)$ of $\mathrm{Nd}^{3}+$ in YSO. An important observation is that the inhomogeneous linewidth of the ions in the cavity is the same as in the bulk material, for both the $0.2 \%\left(\Delta_{\text {inhom }}=16.0 \mathrm{GHz}\right)$ and $0.003 \%$ $(5.9 \mathrm{GHz})$ devices (Supplementary Note 2). A small inhomogeneous linewidth (on the order of $\sim 10 \mathrm{GHz}$ ) is important for scaling to networks of multiple QLMIs (Supplementary Note 3). The cavity resonance was tuned across the $\mathrm{Nd}^{3+} \mathrm{PL}$ line using a gas condensation technique ${ }^{12}$. The spectrograms in Fig. $2 \mathrm{~b}-\mathrm{d}$ show enhancement of the $Y_{1}$ line when the cavity is resonant with it, which is a signature of coupling. The $Y_{2}$ line exhibits negligible enhancement, because its dipole moment is not aligned with the TE cavity polarization ( $D_{1}$ axis of the YSO crystal, Fig. $1 \mathrm{~b}$ ). The spontaneous emission rate enhancement was characterized via lifetime measurements. A pulsed laser at $810 \mathrm{~nm}$ excited fluorescence of the $Y_{1}$ line, which was filtered using a monochromator and detected with a single photon counter (Fig. 2e). From single exponential fits, we calculated a reduction in lifetime from $254 \mu \mathrm{s}$ when the cavity is detuned by $\Delta \lambda=0.3 \mathrm{~nm}$, to $87 \mu \mathrm{s}$ on resonance. Taking into account the branching ratio of the $883 \mathrm{~nm}$ transition ( $\beta \sim 4.5 \%$, see Methods), the reduction in lifetimes corresponds to an ensemble averaged Purcell factor ${ }^{27} F=42$, which agrees well with the estimations that assume a uniform spatial distribution of $\mathrm{Nd}^{3}+$ ions in the resonator (Supplementary Note 4). A single ion positioned at the maximum cavity field would experience a Purcell factor of $\sim 200$. A similar result was obtained in a $0.003 \%$ cavity with lower quality factor (Supplementary Fig. 2 and Supplementary Note 5).

Optical coherence time for cavity-coupled REIs. Coherent and stable optical transitions are essential for QLMIs. We characterized the optical coherence time $T_{2}$ of the 883-nm transition using two-pulse $(\pi / 2-\pi)$ photon echo techniques (Fig. 3a), with an applied magnetic field of $\mathbf{B}=0.5 \mathrm{~T}$ (see Methods). The laser pulses were coupled in and the echoes were collected via the couples when the $0.2 \%$ and $0.003 \%$ cavities were on resonance with the $\mathrm{Nd}$ transition. As only a small sub-ensemble $(<100$ ions) in the cavity was excited, the weak echo signal required 
a

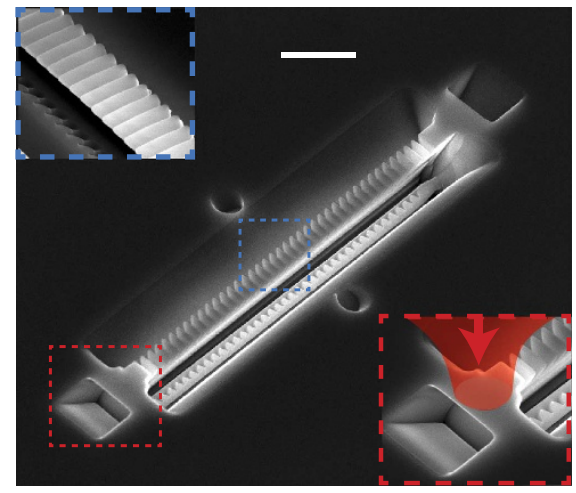

b

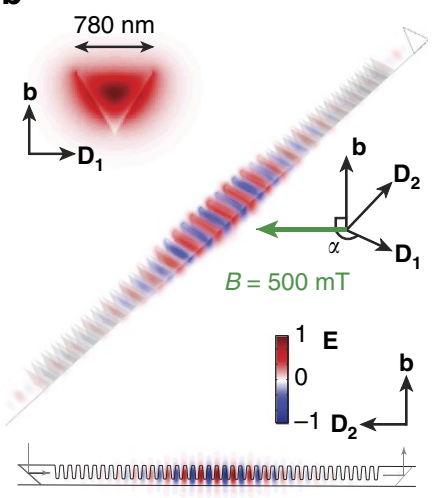

C

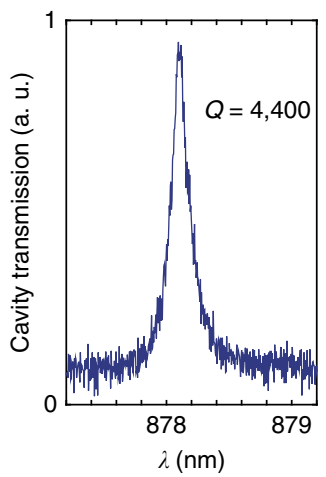

Figure 1 | Photonic crystal nanobeam resonator fabricated in Nd:YSO. (a) Scanning electron microscope image of the device. Scale bar, $2 \mu \mathrm{m}$. The red inset is a zoomed-in view of the $45^{\circ}$ angle-cut coupler that allows vertical coupling of light from a microscope objective. The blue inset shows the grooves forming the photonic crystal. (b) Schematics of the nanobeam resonator with simulated electric field (E along $\mathbf{D}_{1}$ ) profiles of the fundamental TE resonance mode. The TE polarization aligns with the $\mathbf{D}_{1}$ axis of the YSO crystal. A magnetic field of $500 \mathrm{mT}$ is applied in the $\mathbf{D}_{1}-\mathbf{D}_{2}$ plane at an angle of $\alpha=135^{\circ}$ with respect to the $\mathbf{D}_{1}$ axis. (c) Broadband cavity transmission spectrum showing the cavity resonance with quality factor $Q=4,400$.

a

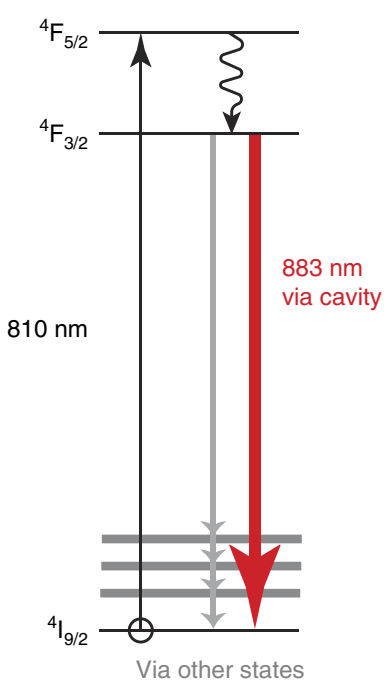

b

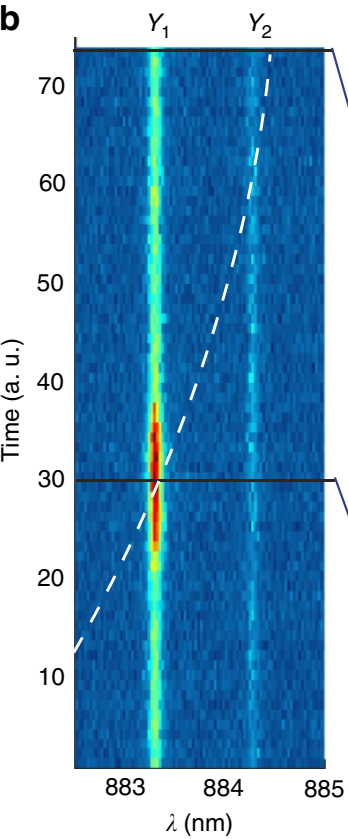

C

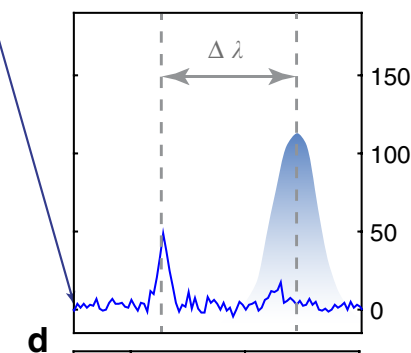

d

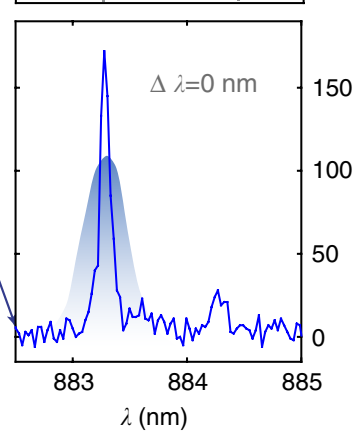

e

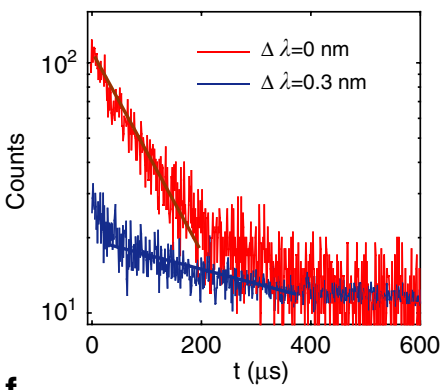

f

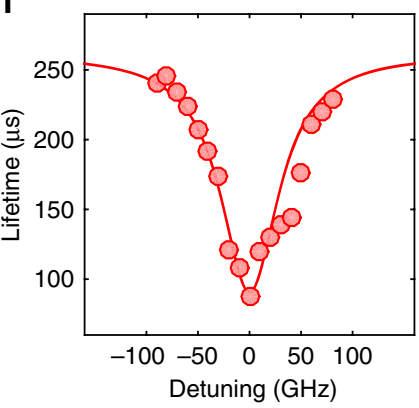

Figure 2 | Purcell-enhanced coupling of $\mathbf{N d}^{\mathbf{3}}{ }^{+}$ions to the YSO cavity mode. (a) Schematic of energy levels for $\mathrm{Nd}^{3+}$ in $\mathrm{YSO}$. Optical excitation at $810 \mathrm{~nm}$ results in $\mathrm{PL}$ at several wavelengths with only the 883-nm transition enhanced by the cavity. (b) Spectrogram showing the $\mathrm{Nd}^{3+} \mathrm{PL}$, while the cavity is tuned across resonance using gas condensation. The dashed line is a guide to the eye indicating the central wavelength of the cavity resonance. The cavity resonance is not visible, because there is no background luminescence to populate the cavity mode. PL spectra in the uncoupled (c) and coupled (d) cases. The cavity resonance was drawn to indicate the cavity location. (e) Lifetime measurements for coupled $\left(\tau^{c}=87 \mu \mathrm{s}, \Delta \lambda=0\right)$ and uncoupled $\left(\tau^{0}=254 \mu \mathrm{s}, \Delta \lambda=0.3 \mathrm{~nm}\right.$ ) cases. (f) Change in lifetime as a function of the cavity detuning, which fits well with the calculation (red curve) using quality factor $Q=4,400,4.5 \%$ branching ratio and field intensity averaged over the mode volume.

detection using single photon counters. A typical echo from the $0.2 \%$ cavity is shown in Fig. $3 \mathrm{~d}$. The echo decays as a function of the $(\pi / 2-\pi)$ time delay $\tau$ are plotted in Fig. 3b together with photon echoes from bulk substrates. Echo intensity decays by $\mathrm{e}$ for delay $\tau_{1 / e}$. For the $0.2 \%$ sample, $T_{2}^{\mathrm{c}}=4 \tau_{1 / \mathrm{e}}=2.8 \pm 0.4 \mu \mathrm{s}$ was measured for the cavity, which shows a good agreement with the bulk value of $T_{2}^{\mathrm{b}}=3.2 \pm 0.4 \mu \mathrm{s}$. For the $0.003 \%$ sample, the echo exhibited two exponential decays. The slower decays give $T_{2}^{\mathrm{b}}=100 \pm 5 \mu \mathrm{s}$ (bulk) and $T_{2}^{\mathrm{c}}=94 \pm 5 \mu \mathrm{s}$ (cavity), which match with values reported in ref. 28 . The fast decays are likely due to the superhyperfine interactions between $\mathrm{Nd}^{3+}$ and its neighbouring yttrium ions, which commonly manifests as modulated echoes decaying faster than $T_{2}$ (ref. 28). No oscillations were observed in Fig. $3 \mathrm{a}$ because of the fast modulation frequency $(\sim 1 \mathrm{MHz})$ due to the strong magnetic field. Oscillations of echoes for the initial $10 \mu$ s delay were observed when the $\mathbf{B}$ field was reduced to $<100 \mathrm{mT}$. In addition, changes in the $T_{2}$ values were not observed as the excitation power was varied, which indicates the measurement was not significantly affected by instantaneous spectral diffusion. In sum, the good agreement on $T_{2}$ between the cavity and bulk confirms that the optical coherence property of $\mathrm{Nd}^{3+}$ ions is not affected by the nanofabrication. For higher Purcell factors, the $T_{2}$ in cavities should decrease owing to the $T_{2} \leq 2 T_{1}$ limit and would become 
a

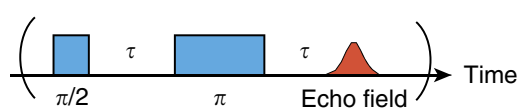

b

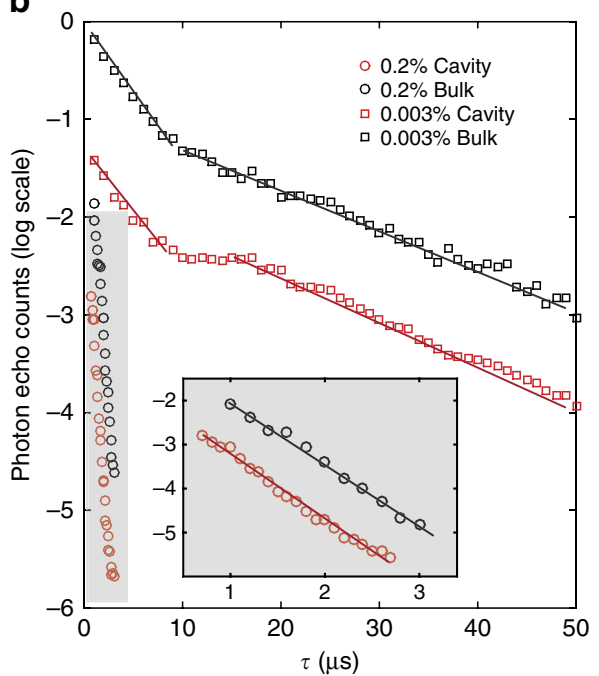

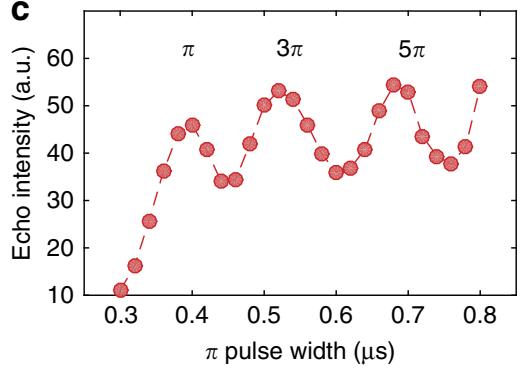

d 80

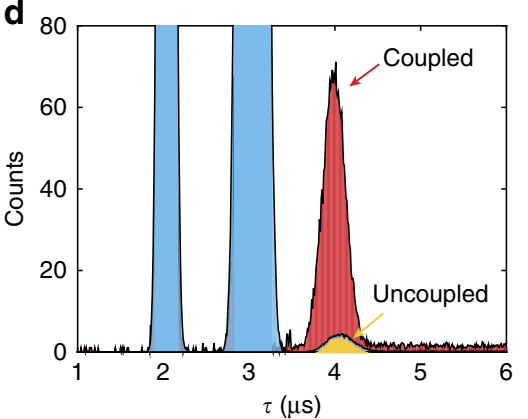

Figure 3 | Photon echo measurements from an ensemble of $\mathbf{N d}^{\mathbf{3}+}$ ions in the cavity. (a) Two-pulse photon echo sequence $(\pi / 2-\pi)$ used to measure $T_{2}$. (b) Two-pulse photon echo decays measured in both the cavity (red) and the bulk (black) samples with two different doping concentrations. The inset plots the echo decays measured with a $0.2 \%$ doped sample. (c) Oscillation of echo intensity with increasing width of the $\pi$ rephasing pulse. The periodic signal reveals the ensemble averaged Rabi frequency of the coupled ions. The ideal $\pi$ pulse duration for the input power was $0.4 \mu$ s. (d) Enhanced photon echo intensity (by $\sim 12$-fold) when the cavity is coupled, compared with the uncoupled case (cavity detuned by $\Delta \lambda=15 \mathrm{~nm}$ so that the transition is outside the photonic bandgap).

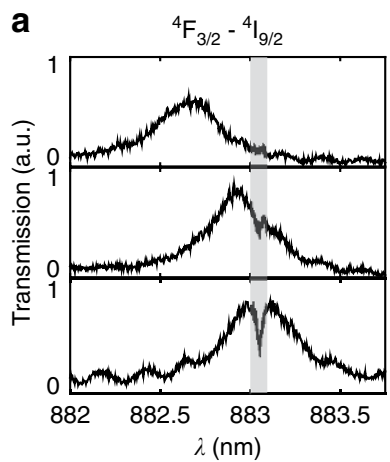

b

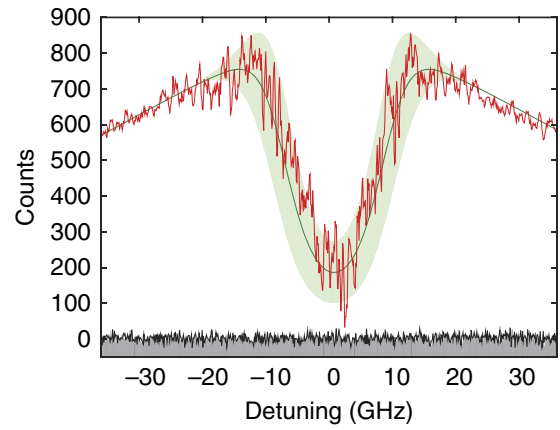

C

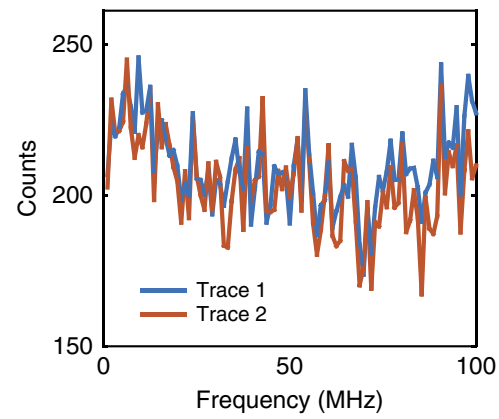

Figure 4 | Control of cavity transmission and observation of SFS. (a) Broadband transmission spectra as the cavity is tuned to the $883 \mathrm{~nm} \mathrm{Nd}$ transition. A dip is observed when the two are on resonance. The negligible dip at far detunings confirms that this effect is not due to absorption, but quantum interference between the intracavity field and the ions. (b) High-resolution transmission spectrum (red curve) obtained by scanning a narrow linewidth ( $\sim 20 \mathrm{kHz}$ ) Ti:Sapphire laser over the inhomogeneous line. The green curve is the fit using parameters: $\bar{g}=2 \pi \times 6 \mathrm{MHz}, \Gamma_{\mathrm{h}}=2 \pi \times 100 \mathrm{kHz}$,

$\Gamma_{\text {inhom }}=16.0 \mathrm{GHz}$, and assuming a Gaussian ion density distribution. The green shaded region is the estimated fluctuation in the transmitted laser intensity caused by $\sqrt{N}$ statistical variations of the ion density. Large fluctuations are expected, because the density $N$ is low (few tens), which agrees with the measurement. The fluctuation within the inhomogeneous linewidth is noticeably larger than that at far detunings $(>25 \mathrm{GHz})$ and the technical background noise (grey area), confirming that they are caused by SFS of the ion spectral density. (c) Two traces of the transmitted intensities over the same $100 \mathrm{MHz}$ bandwidth near zero detuning at different times. The high degree of correlation confirms the static and repeatable nature of SFS.

smaller than the bulk value. This regime is not reached here, because the Purcell enhanced $2 T_{1}$ is not smaller than $T_{2}^{\mathrm{b}}$.

The observation of photon echoes demonstrates coherent optical control of the quantum state of cavity-coupled ions. This control was further extended by varying the $\pi$ pulse duration and observing Rabi oscillations in the echo intensities as shown in Fig. 3c. A Rabi frequency of $\sim 6 \mathrm{MHz}$ is estimated. The same oscillation was not observed in the bulk. For the coupled laser power, the optimal $\pi$ pulse duration is $0.4 \mu \mathrm{s}$. The oscillations are not visible for pulse duration $<0.3 \mu \mathrm{s}$, because of the limited rise/fall times ( $\sim 200 \mathrm{~ns})$ of the pulse-generating setup (Methods).
A 12-fold increase in the echo intensity is observed in the cavity-coupled case compared with the uncoupled case (that is, detuning $\Delta \lambda=15 \mathrm{~nm}$ ) as shown in Fig. $3 \mathrm{~d}$. This enhancement can be attributed to a combination of several effects: the higher atomic absorption rate through the Purcell effect ${ }^{29}$, stronger intracavity field intensity and high echo collection efficiency as the ions emit dominantly into the cavity mode. The spectral diffusion of the coupled ions using three-pulse photon echoes was also investigated. The homogeneous linewidths were broadened at rates of $6.1 \mathrm{kHz} \mu \mathrm{s}^{-1}$ for the $0.2 \%$ doped cavity and $380 \mathrm{~Hz}^{-1}$ for the $0.003 \%$ cavity. These slow spectral 
diffusions permit repeated optical addressing of the ions for $10 \mathrm{~s}$ of $\mu$ s (Supplementary Fig. 3 and Supplementary Note 6).

Dipole-induced transparency and statistical fine structure. QLMIs require efficient interactions between atoms and photons, which is why quantum memories use long atomic clouds or doped crystals to achieve large optical depth. One key advantage provided by nanoresonators is that efficient atom-photon interaction can be achieved in a small volume with only a handful of ions. This is readily observable in our system, where the $\mathrm{Nd}^{3+}$ ions coherently interact with the intracavity field and control its transmission via dipole-induced transparency ${ }^{30}$. With the cavity tuned to $883 \mathrm{~nm}$, the cavity transmission was probed using broadband light and a dip was observed at resonance (Fig. 4a). The depth of the dip depends on the collective coupling cooperativity $\eta=4 N \bar{g}^{2} /\left(\kappa \Gamma_{\mathrm{h}}\right)$, where $\bar{g}$ is the ensemble averaged coupling strength, $\kappa$ is the cavity full linewidth, $\Gamma_{\mathrm{h}}$ is the $\mathrm{Nd}^{3+}$ homogeneous linewidth and $N$ is the number of ions per $\Gamma_{\mathrm{h}}$. Considering an empirical collective dipole-cavity coupling model, the normalized cavity transmission in the presence of unsaturated resonant ions is,

$$
T=\left|\frac{\kappa}{i \Delta+\kappa+4 N \bar{g}^{2} / \Gamma_{\mathrm{h}}}\right|^{2},
$$

which simplifies to $T=(1+\eta)^{-2}$ for zero detuning ${ }^{31}$. The cavity transmission can be controlled by varying the probe light power and observing the saturation of the ions at increasing intracavity photon number (Supplementary Fig. 4 and Supplementary Note 7). The saturation photon number in the nanocavity was measured to be $\left\langle n_{\text {cav }}\right\rangle=2 \times 10^{-5}$.

To better resolve the spectrum, a narrow $(\sim 20 \mathrm{kHz})$ Ti:Sapphire laser was scanned across the resonance (see Methods) to give the transmitted signals shown in Fig. 4b,c. A 75\% decrease in transmission was measured at zero detuning, which corresponds to a collective cooperativity $\eta \sim 1.2$. Fitting using a Gaussian spectral density distribution (green line) with measured parameters $\bar{g}=2 \pi \times 6 \mathrm{MHz}, \Gamma_{\mathrm{h}}=2 \pi \times 100 \mathrm{kHz}$ and $\Gamma_{\text {inhom }}=16.0 \mathrm{GHz}$ gives a peak ion density of $N \approx 53$. Because of the statistical fine structure (SFS) of the inhomogeneously broadened line, a variation in the transmitted intensity $\left(\langle\delta T\rangle^{2} \sim \frac{d^{2} T}{d N^{2}}\langle\delta N\rangle^{2}\right)$, owing to $\delta N=\sqrt{N}$ fluctuations in the ion spectral density is expected ${ }^{32}$. This expected variation is represented in Fig. $4 \mathrm{~b}$ by the green shaded region and shows good agreement with that of the measured signal. This variation within the inhomogeneously broadened line, on which the statistical fluctuations of the ion spectral density are imprinted, is significantly larger than the spectrometer technical noise (grey area) and laser shot noise at far detunings $(>25 \mathrm{GHz})$, thus confirming that the static SFS in spectral density $N(\Delta \lambda)$ is probed. Two traces of the laser scan over the same $100 \mathrm{MHz}$ bandwidth near zero detuning at different times are shown in Fig. 4c. The high degree of correlation reflects the static and repeatable nature of SFS. Notably, the current platform would allow detection and control of a single ion coupled to the cavity if $N \ll 1$ and the laser linewidth were narrower than $\Gamma_{h}$ (Supplementary Fig. 5 and Supplementary Note 8).

\section{Discussion}

The results reported in this paper (long optical coherence time, small inhomogeneous broadening, enhanced coherent optical control and resonant probing of cavity-coupled REIs) demonstrate REI-based nanophotonics as a promising approach for robust and scalable quantum photonic networks integrating memories and single REI qubits. Single photon rates exceeding $1 \mathrm{MHz}$ can be achieved with single REIs in nanocavities with
$Q / V \sim 10^{4}-10^{5}$ ( $V$ is normalized to $\left.(\lambda / n)^{3}\right)$ and the inhomogeneous broadening allows for frequency multiplexing of multiple REIs. To use the interface as an optical quantum memory, efficient optical pumping into the long-lived Zeeman level needs to be demonstrated. Bulk REI quantum memories already boast high storage efficiency ${ }^{33}$ with multi-mode capacity ${ }^{28}$. Their implementations in our nanophotonic platform open the possibility of multiplexed systems for on-chip quantum repeaters. For Nd, high-fidelity storage of entanglement based on atomic frequency comb has been demonstrated ${ }^{34,35}$. With cavity impedance matching ${ }^{29}$, unit storage efficiency is achievable with a mesoscopic ensemble of cavity-coupled ions. Meanwhile, long-lived nuclear spin coherence of $9 \mathrm{~ms}$ in ${ }^{145} \mathrm{Nd}$ (ref. 36) bodes well for spin-wave quantum memories using our nanoresonators. These devices can be further coupled to superconducting or optomechanical devices, to enable hybrid quantum systems 4 . Furthermore, the technology can be readily transferred to other wavelengths, such as $1.5 \mu \mathrm{m}$ for telecom quantum memories using $\mathrm{Er}^{3+}$ :YSO or $580 \mathrm{~nm}$ for long-haul quantum hard drives using $\mathrm{Eu}^{3+}$ :YSO (ref. 16).

\section{Methods}

YSO nanoresonator design and fabrication. The nanobeam has an equilateral triangular cross-section with each side of $780 \mathrm{~nm}$. This geometry allows a circular fundamental mode field that can be efficiently coupled with a free space laser beam. The cavity is formed by 40 equally spaced grooves of lattice constant $340 \mathrm{~nm}$ on the nanobeam, except for a defect introduced at the centre by perturbing the lattice constant. The depth of the grooves is $65 \%$ of the beam height. The triangular nanobeam resonator was fabricated using focused ion beam milling followed by wet etching of $\mathrm{Ga}^{+}$contaminated YSO in diluted (10\%) hydrochloric acid. An ion beam of $20 \mathrm{kV}, 0.2 \mathrm{nA}$ was used to fabricate the suspended nanobeam waveguide by milling at $30^{\circ}$ angle with respect to the crystal surface normal. A small ion beam of $23 \mathrm{pA}$ was then used to accurately pattern the grooves on top of the nanobeam. Limited by the finite width of the focused ion beam, the side walls of the grooves in the actual device were not vertical, but had an angle of $6^{\circ}$. This leads to a degraded theoretical $Q$ of $5.0 \times 10^{4}$. We were able to reproducibly fabricate arrays of resonators (up to six) in a batch (Supplementary Fig. 1), with all the devices measuring resonances close to $883 \mathrm{~nm}$ and quality factors varying from 1,100 to 10,000 .

Experimental setup for the photon echo measurements. A 500-mT external magnetic field was applied at $\alpha=135^{\circ}$ relative to the crystal $D_{1}$ axis using a pair of permanent magnets (see Fig. 1b). The $\pi / 2$ and $\pi$ Gaussian pulses were generated by amplitude-modulating the Ti:Sapphire laser with two acousto-optic modulators (AOMs) in series, with each in a double-pass configuration. The two pulse widths were 250 and $400 \mathrm{~ns}$ at a repetition rate of $1 \mathrm{kHz}$. The average (peak) power of the excitation pulses was $210 \mathrm{nW}(320 \mu \mathrm{W})$ measured after the objective lens. The extinction ratio between the on and off level of the pulses was $\sim 120 \mathrm{~dB}$, ensuring sufficient signal-to-noise ratio for detecting echo photons using a Si single-photon counter (Perkin Elmer SPCM). A third shutter AOM in single-pass configuration was inserted just before the photon counter to block the strong excitation pulses from saturating the detector. The extinction ratio of this shutter AOM was $30 \mathrm{~dB}$.

High-resolution laser spectroscopy on cavity-coupled $\mathbf{N d}^{\mathbf{3}}+$ ions. For the cavity transmission experiments, the Ti:Sapphire laser (M Squared SolsTiS) was continuously scanned at a rate of $10 \mathrm{MHz}$ per second. The high-sensitivity charge-coupled device camera in the spectrometer (Princeton Instruments PIXIS) registers the transmitted photon counts intermittently at an adjustable frame rate (frames per second (fps)) with an exposure time $0.01 \mathrm{~s}$ for each frame. Therefore, one exposure corresponds to a spectral width of $10 \mathrm{MHz} \times 0.01=100 \mathrm{kHz}$ scanned by the laser, which is equal to the homogeneous linewidth of the $0.2 \%$ doped sample. The long-term drift of the laser is $10 \mathrm{MHz}$ per hour, so the drift during each exposure should be inconsequential. Each data point in Fig. 4b,c represents the photon count collected in one camera exposure, corresponding to the signal contributed by the ions within one homogeneous linewidth. The frame rate was $0.1 \mathrm{fps}$ for the coarser scan in Fig. 4b, corresponding to a spectral interval of $100 \mathrm{MHz}$ between two adjacent data points. The frame rate was $8.2 \mathrm{fps}$ for the fine scan in Fig. $4 c$, with a spectral interval $\sim 1 \mathrm{MHz}$. Each data point was obtained with one scan. Several scans at different spectral regions were performed and stitched together to cover the entire bandwidth in Fig. $4 \mathrm{~b}$.

Estimation of the branching ratio. The measured optical depth of a $15-\mu \mathrm{m}$-long nanobeam resonator at $3.8 \mathrm{~K}$ was $d=0.1$, from which we deduce an oscillator strength of $f=6.5 \times 10^{-7}$ and a spontaneous emission rate of this transition to be 
$\gamma_{883}=1 / \tau_{883}=1 / 5.6 \mathrm{~ms}$ (ref. 24). With a measured bulk medium lifetime $\tau_{0}=250 \mu \mathrm{s}$, the branching ratio was thus estimated to be $\tau_{0} / \tau_{883} \approx 4.5 \%$.

\section{References}

1. Kimble, H. J. The quantum internet. Nature 453, 1023-1030 (2008).

2. Kómár, P. et al. A quantum network of clocks. Nat. Phys. 10, 582-587 (2014).

3. Chen, W. et al. All-optical switch and transistor gated by one stored photon. Science 314, 768-770 (2013).

4. Williamson, L. A., Chen, Y.-H. \& Longdell, J. J. Magneto-optic modulator with unit quantum efficiency. Phys. Rev. Lett. 113, 203601 (2014).

5. Andrews, R. W. et al. Bidirectional and efficient conversion between microwave and optical light. Nat. Phys. 10, 321-326 (2014).

6. Michler, P. (ed.) Single Quantum Dots (Springer Berlin Heidelberg, 2009).

7. Aharonovich, I. \& Neu, E. Diamond nanophotonics. Adv. Optical Mater. 2, 911-928 (2014).

8. Bernien, H. et al. Heralded entanglement between solid-state qubits separated by three metres. Nature 497, 86-90 (2013).

9. Press, D., Ladd, T. D., Zhang, B. \& Yamamoto, Y. Complete quantum control of a single quantum dot spin using ultrafast optical pulses. Nature 456, 218-221 (2008).

10. De Greve, K. et al. Quantum-dot spin-photon entanglement via frequency downconversion to telecom wavelength. Nature 491, 421-425 (2012).

11. Englund, D. et al. Controlling cavity reflectivity with a single quantum dot. Nature 450, 857-861 (2007).

12. Faraon, A., Barclay, P. E., Santori, C., Fu, K. C. \& Beausoleil, R. G. Resonant enhancement of the zero-phonon emission from a colour centre in a diamond cavity. Nat. Photon. 5, 301-305 (2011).

13. Li, L. et al. Coherent spin control of a nanocavity-enhanced qubit in diamond. Nat. Commun. 6, 6173 (2015).

14. Faraon, A., Santori, C., Huang, Z., Acosta, V. M. \& Beausoleil, R. G. Coupling of nitrogen-vacancy centers to photonic crystal cavities in monocrystalline diamond. Phys. Rev. Lett. 109, 033604 (2012).

15. Thiel, C., Böttger, T. \& Cone, R. Rare-earth-doped materials for applications in quantum information storage and signal processing. J. Luminesc. 131, 353-361 (2001).

16. Zhong, M. et al. Optically addressable nuclear spins in a solid with a six-hour coherence time. Nature 517, 177-180 (2015).

17. Sun, Y., Thiel, C. W., Cone, R. L., Equall, R. W. \& Hutcheson, R. L. Recent progress in developing new rare earth materials for hole burning and coherent transient applications. J. Lumin. 98, 281-287 (2002).

18. Lvovsky, A. I., Sanders, B. C. \& Tittel, W. Optical quantum memory. Nat. Photon. 3, 706-714 (2009)

19. Tittel, W. et al. Photon-echo quantum memory in solid state systems. Laser Photon. Rev. 4, 244-267 (2010).

20. Saglamyurek, E. et al. Broadband waveguide quantum memory for entangled photons. Nature 469, 512-515 (2011).

21. Marzban, S., Bartholomew, J. G., Madden, S., Vu, K. \& Sellars, M. J. Observation of photon echoes from evanescently coupled rare-earth ions in a planar waveguide. Phys. Rev. Lett. 115, 013601 (2015).

22. Kolesov, R. et al. Optical detection of a single rare-earth ion in a crystal. Nat. Commun. 3, 1029 (2012).

23. Utikal, T. et al. Spectroscopic detection and state preparation of a single praseodymium ion in a crystal. Nat. Commun. 5, 3627 (2014).

24. McAuslan, D. L. \& Longdell, J. J. Cavity QED using rare-earth-metal-ion dopants in monolithic resonators: what you can do with a weak oscillator. Phys. Rev. A 80, 062307 (2009).

25. Bayn, I., Meyler, B., Salzman, J. \& Kalish, R. Triangular nanobeam photonic cavities in single-crystal diamond. N. J. Phys. 13, 025018 (2011).
26. Oskooi, A. F. et al. MEEP: a flexible free-software package for electromagnetic simulations by the FDTD method. Comput. Phys. Commun. 181, 687-702 (2010).

27. Purcell, E. M. Spontaneous emission probabilities at radio frequencies. Phys Rev. 69, 681 (1946).

28. Usmani, I., Afzelius, M., de Riedmatten, H. \& Gisin, N. Mapping multiple photonic qubits into and out of one solid-state atomic ensemble. Nat. Commun. 1, 12 (2010).

29. Afzelius, M. \& Simon, C. Impedance-matched cavity quantum memory. Phys Rev. A 82, 022310 (2010)

30. Waks, E. \& Vucković, J. Dipole induced transparency in drop-filter cavity-waveguide systems. Phys. Rev. Lett. 96, 153601 (2006).

31. Thompson, J. D. et al. Coupling a single trapped atom to a nanoscale optical cavity. Science 340, 1202-1205 (2013).

32. Moerner, W. E. \& Carter, T. P. Statistical fine structure of inhomogeneously broadened absorption lines. Phys. Rev. Lett. 59, 2705 (1987).

33. Hedges, M. P., Longdell, J. J., Li, Y. \& Sellars, M. J. Efficient quantum memory for light. Nature 465, 1052-1056 (2010).

34. Clausen, C. et al. Quantum storage of photonic entanglement in a crystal. Nature 469, 508-511 (2011).

35. Bussières, F. et al. Quantum teleportation from a telecom-wavelength photon to a solid-state quantum memory. Nat. Photon. 8, 775-778 (2014).

36. Wolfowicz, G. et al. Coherent storage of microwave excitations in rare earth nuclear spins. Phys. Rev. Lett. 114, 170503 (2015).

\section{Acknowledgements}

We gratefully acknowledge the contributions of Alexander E. Hartz. This work was funded by California Institute of Technology (Caltech) and National Science Foundation (NSF) CAREER award number 1454607. Equipment funding was also provided by the Institute of Quantum Information and Matter (IQIM), an NSF Physics Frontiers Center with support of the Moore Foundation. The device nanofabrication was performed in the Kavli Nanoscience Institute at Caltech.

\section{Author contributions}

A.F. and T.Z. conceived the experiments. T.Z. and E.M. performed the simulations and T.Z. fabricated the devices. T.Z. and J.M.K. performed the measurements and analysed the data. T.Z. and A.F. wrote the manuscript with input from all authors.

\section{Additional information}

Supplementary Information accompanies this paper at http://www.nature.com/ naturecommunications

Competing financial interests: The authors declare no competing financial interests

Reprints and permission information is available online at http://npg.nature.com/ reprintsandpermissions/

How to cite this article: Zhong, T. et al. Nanophotonic coherent light-matter interfaces based on rare-earth-doped crystals. Nat. Commun. 6:8206 doi: 10.1038/ncomms9206 (2015).

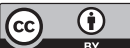

This work is licensed under a Creative Commons Attribution 4.0 International License. The images or other third party material in this article are included in the article's Creative Commons license, unless indicated otherwise in the credit line; if the material is not included under the Creative Commons license, users will need to obtain permission from the license holder to reproduce the material. To view a copy of this license, visit http://creativecommons.org/licenses/by/4.0/ 